\title{
Periodontal Disease: A Veiled Epidemic with Nascent Public Health Approach
}

\author{
Siddharthan Selvaraj* \\ Faculty of Medicine, Medical Campus, Universiti Sultan Zainal Abidin, Kuala Terengganu, Terengganu, Malaysia \\ *Corresponding Author: Siddharthan Selvaraj, Faculty of Medicine, Medical Campus, Universiti Sultan Zainal Abidin, Kuala Tereng- \\ ganu, Terengganu, Malaysia.
}

Received: January 24, 2022; Published: January 31, 2022

Periodontal disease is an inflammatory condition which affects and damages the tissues that supports and surround the tooth, it is seen among 15 to $20 \%$ of population around the globe [1]. Periodontal disease can also be considered as epidemic based on its prevalence. To add upon, severity of this oral condition haven't been spoken for years [2]. It has been reported that individuals with periodontal disease are more vulnerable to get systemic conditions like diabetes mellitus and cardiovascular disorders [3]. In spite of the fact that periodontal disease plays a significant role in an individual's self-esteem, maintenance of oral hygiene still been ignored. $[4,5]$. Periodontal disease is one of the serious oral conditions that can be prevented when found at initial stage. Periodontal disease begins as gingivitis and transforms into periodontitis if undiagnosed at initial stage [6]. Periodontitis leads to loss of tooth, masticatory complication that results in lack of nutritional intake of an individual and has impact on quality of life of a person [7, 8]. Although periodontal disease is an inflammatory condition and seen among every nation in the globe, public health model implementation for controlling and preventing periodontal disease is unreal [9]. Public health approach to prevent from a disease and promote an individual's health can be achieved by assessment of health status, development of policy and assurance - evaluating the effectiveness of the approach [10].

Petersen and Ogawa stated that every country should set up a monitoring institution to analyse the advancement that is been taking place on periodontal disease control and oral health promotion [11]. Even after a decade, the public health approach of periodontal disease on control and prevention remains stagnant in assessment stage. Establishment of public health strategy to prevent periodontal disease and enhancing the oral hygiene stays in a budding stage. Hence, there is a need to create awareness on relationship between periodontal disease with health and wellbeing of an individual to portray periodontal disease as a serious public health issue [9].

At individual level, promotion and prevention of periodontal disease is achievable. On the flip side, we are still lacking with strategies to implement promotion and prevention of periodontal condition at community level. By utilizing modern data science and technological tools with public health informatics we can get a desirable outcome, it paves way for a better policy towards public health care. Secondly, it can also assist in promoting periodontal hygiene of an individual, identifying approaches that would help in integrating overall health and periodontal health care. Finally, to enhance literacy towards oral health [9] as an individual's oral health knowledge, attitude and behavior plays a vital role in maintaining oral hygiene $[12,13]$.

Despite the fact that prevalence of periodontal disease is growing high due to the ageing population, there is a vacuum in plans and lack of importance been given to enhance the periodontal health. Giving equal importance, implementing, and monitoring the collaborative public health approach between general and oral health might be an ideal way to tackle periodontal disease. At this moment, public health care approach for periodontal disease is very limited. Keeping in mind on previous studies, it is the right moment to implement effective oral health policies that helps to monitor the periodontal disease control and promotion of periodontal hygiene which would pave a way to explore the disease prevalence and preventive measures in a broader way to bring it to limelight as a serious non communicable disease.

Citation: Siddharthan Selvaraj. “Periodontal Disease: A Veiled Epidemic with Nascent Public Health Approach”. Medicon Dental Sciences 1.1 (2022): 20-21. 


\section{References}

1. Nazir M., et al. "Global prevalence of periodontal disease and lack of its surveillance". The Scientific World Journal (2020).

2. Nocini R., et al. "Periodontal disease: The portrait of an epidemic". J. Public Health Emerg 4.10 (2020).

3. Assiry AA., et al. "Periodontal disease among Saudi Arabia and South Asian developing nations". Int J Pharm Res 13.2 (2021): 565-70.

4. Siddharthan, S., et al. “Oral health and services in India”. Int J Pharm Res 13.1 (2021): 3786-3790.

5. Selvaraj S., et al. "Assessment on Oral Health Knowledge, Attitude, and Behaviour and its Association with Sociodemographic and Habitual Factors of South Indian Population". Pesquisa Brasileira em Odontopediatria e Clínica Integrada 21 (2021).

6. Siddharthan S., et al. "Periodontal diseaseand COVID 19". Journal of Pharmaceutical Research International (2020): 88-91.

7. Selvaraj S., et al. “Demographic and Habitual Factors of Periodontal Disease among South Indian Adults”. International journal of environmental research and public health 18.15 (2021): 7910.

8. Selvaraj S., et al. "Effect of periodontal health in marital life". Research Journal of Pharmacy and Technology 14.8 (2021): 44634465.

9. Janakiram., et al. "A public health approach for prevention of periodontal disease". Periodontology 84.1 (2020): 202-214.

10. Institute of Medicine (US) Committee for the Study of the Future of Public Health. The Future of Public Health. Washington, DC: National Academies Press (1988).

11. Petersen PE and Ogawa H. "Strengthening the prevention of periodontal disease: the WHO approach". J Periodontol 76.12 (2005): 2187-2193.

12. Selvaraj S., et al. "Development and Validation of Oral Health Knowledge, Attitude and Behavior Questionnaire among Indian Adults". Medicina 58.1 (2022): 68.

13. Selvaraj S., et al. “Confirmatory Factor Analysis of Knowledge, Attitude, and Behaviour Questionnaire towards Oral Health among Indian Adults". Journal of Personalized Medicine 11.4 (2021).

Volume 1 Issue 1 February 2022

(C) All rights are reserved by Siddharthan Selvaraj. 